\title{
Congenital Rubella Syndrome in The African Region - Data from Sentinel Surveillance
}

\author{
Balcha Masresha ${ }^{1 *}$, Messeret Shibeshi' ${ }^{2}$ Reinhard Kaiser ${ }^{4}$, Richard Luce ${ }^{3}$, Regis Katsande', Richard Mihigo' \\ 'WHO Regional Office for Africa. Brazzaville, Congo \\ ${ }^{2}$ WHO Inter-country Support Team for East and Southern Africa. Harare, Zimbabwe \\ ${ }^{3}$ WHO Inter-country Support Team for Central Africa. Libreville, Gabon \\ ${ }^{4}$ Formerly with the WHO Inter-country Support Team for East and Southern Africa. Harare, Zimbabwe
}

Article Info

\section{Article Notes}

Published: August 02, 2018

\section{${ }^{*}$ Correspondence:}

Dr. Balcha G Masresha, WHO Regional Office for Africa, Brazzaville, Congo; Telephone No: +263 77503 5369;

Email: masreshab@who.int.

() 2018 Masresha BG. This article is distributed under the terms of the Creative Commons Attribution 4.0 International License.

\section{Keywords:}

Congenital Rubella Syndrome

Sentinel Surveillance

African Region

\section{ABSTRACT}

Introduction: Rubella is a mild febrile rash illness caused by the rubella virus. The most serious consequence of rubella is congenital rubella syndrome (CRS), which occurs if the primary rubella infection occurs during early pregnancy, with subsequent infection of the placenta and the developing fetus.

Methods: WHO supported countries to set up sentinel surveillance for CRS using standard case definitions, protocols, and case classification scheme. This descriptive analysis summarises the data from 5 countries which have been regularly reporting.

Results : A total of 383 suspected cases of CRS were notified from the 5 countries as of December 2016, of which 52 cases were laboratory confirmed and 67 were confirmed on clinical grounds.

The majority (43\%) of confirmed CRS cases were in the age group $6-11$ months. The most common major clinical manifestation (Group A) among the confirmed cases is congenital heart disease $(72 \%)$ followed by cataracts $(32 \%)$ and glaucoma (10\%).

Discussion and conclusions: The number of years of reporting from these sentinel sites is too short to describe trends in CRS occurrence across the years. However, the limited surveillance data has yielded comparable information with other developing countries prior to introduction of rubella vaccine. As more countries introduce rubella vaccine into their immunisation programs, there is a need to ensure that all rubella outbreaks are thoroughly investigated and documented, to expand sentinel surveillance for CRS in more countries in the Region, and to complement this with retrospective record reviews for CRS cases in selected countries.

\section{Introduction}

Rubella is a mild febrile rash illness caused by the rubella virus. In childhood, the illness is characterized by a transient, erythematous rash, low grade fever, post-auricular and sub-occipital lymphadenopathy, sore throat, red eyes, headache, malaise and anorexia $^{1,2}$.

The most serious consequence of rubella is congenital rubella syndrome (CRS), which occurs if the primary rubella infection occurs during early pregnancy, with subsequent infection of the placenta and the developing fetus. The risk for congenital defects has been estimated at $90 \%$ for maternal infection before 11 weeks of gestation. Nerve deafness is the single most common finding among infants with CRS. Unilateral or bilateral cataracts are the most serious eye finding, occurring in about a third of infants. Patent ductus arteriosus is the most frequently reported cardiac defect $t^{1,3}$. 
Diagnosis of rubella or CRS requires laboratory confirmation. The detection of rubella specific IgM is the most widely available method for routine laboratory diagnosis. In CRS cases, IgM antibodies are sometimes found for up to 1 year after birth, and persistence of IgG antibodies beyond 6 months of age has been detected. In addition, rubella virus can be isolated from nasopharyngeal, blood, throat, urine, and cerebrospinal fluid specimens from rubella and CRS cases $^{4,5}$.

Rubella is endemic in countries in the African Region, with a large proportion of cases occurring in children less than 15 years of age ${ }^{6-10}$. However, susceptibility to rubella is known to occur in adults as well. In Burkina Faso, a serosurvey among 341 pregnant women found an overall seropositivity rate of $95.0 \%$ for rubella-specific IgG antibodies indicating a continuous transmission of endemic rubella virus in Burkina Faso, posing a threat to non-immune pregnant women ${ }^{11}$. Similar results have been documented from other studies as well ${ }^{12}$.

The WHO African region has set a goal to eliminate measles by $2020^{13}$. However, currently, there is no Regional goal for rubella/ CRS elimination. WHO recommends that countries should take the opportunity offered by accelerated measles control and elimination activities to introduce rubella vaccine, and incorporate rubella/ CRS control or elimination activities into their immunisation programs $s^{13,14,15}$. GAVI support is available to eligible countries to introduce rubella vaccine as per the WHO guidelines ${ }^{16}$. As of September 2017, only 20 of the 47 countries in the African Region have introduced rubella containing vaccine in their immunisation schedules ${ }^{17}$.

One of the reasons for the low uptake of rubella vaccine in the African Region is the lack of information on disease burden. The WHO African Region is currently supporting countries to generate some information on rubella incidence as part of the measles case based surveillance system. Epidemiological information and blood specimens are collected from persons with suspected measles cases and tested for measles-specific immunoglobulin M (IgM) antibody using standard enzyme-linked immunosorbent assay (ELISA). If results become negative for measles IgM, sera are subsequently tested for rubella-specific IgM antibody using standard ELISA testing ${ }^{5,18}$.

In the African Region, the number of CRS cases reported through the annual aggregate reporting system to the WHO comes to a total of 242 cases in the 10 years period between 2007 - 2016, ranging from 0 cases reported in the entire Region in 2008 and 2011 to 78 cases in 2015. During these years, no more than 4 countries reported at least 1 case of CRS per year, indicating the huge gaps in the reporting system ${ }^{19}$.

The incidence of CRS is underestimated in surveillance systems because of the fact that CRS is not a common condition, the subtle nature of some of the clinical manifestations, the technical difficulty in diagnosing the condition, the lack of an organized surveillance system, and more importantly the lack of facilities to confirm the cases by laboratory means. In the African Region, it is estimated that 38,712 cases occurred in 2010, while the global estimate was $105,391^{20}$. In 2013, a study estimated the incidence of CRS to be $69 / 100,000$ live births in DRC, corresponding to 2,886 infants $(95 \%$ CI 342,6395$)$ born with CRS per year ${ }^{21}$.

WHO supported 11 countries to set up CRS sentinel surveillance between 2011 and 2016 in order to better document the occurrence of CRS so as to help in the initial decision to introduce rubella vaccine and also to help in monitoring impact following introduction. The sentinel surveillance sites from 5 of these countries (Zimbabwe, Tanzania, Rwanda, Burkina Faso, Zambia) have been regularly reporting to the WHO. Burkina Faso, Zimbabwe and Tanzania introduced rubella vaccine in their routine immunisation program following catch-up supplementary immunization activities in 2015, while Rwanda and Zambia introduced it in 2013 and 2016 respectively.

\section{Methods}

Countriesidentified sentinelsurveillancesites, especially tertiary care and specialty centers, based on the presence of clinical expertise and the likelihood of seeing infants with suspected CRS. Following the detection of a suspected case in the sentinel sites, surveillance focal persons identified from among the clinicians on the site examine the child and collect relevant demographic and clinical information using a standardised case investigation form. Serum specimen is collected for laboratory testing for rubella IgM, and laboratory testing is done in the accredited national measles and rubella serological laboratory using standard ELISA testing as per the existing protocols $s^{4,5}$. WHO AFRO standard case definitions and algorithm is utilised for the classification of CRS cases $^{18,22}$.

A suspected case of CRS is any infant less than one year of age in whom a health worker suspects CRS, either from maternal history of suspected or confirmed rubella during pregnancy, or when the infant presents with one or more of the following: heart disease; suspicion of deafness; eye signs including any of these- cataract, diminished vision, nystagmus, squint, micropthalmos, congenital glaucoma.

A clinically-confirmed case is one in which a qualified physician detects in the infant

- two major clinical manifestations (group A): OR

- one clinical manifestation from group A and one of the minor manifestations from group $\mathrm{B}$ :

Group A clinical signs include: Cataract(s), congenital glaucoma; congenital heart disease; hearing impairment; pigmentary retinopathy. Group B clinical signs include: Purpura; splenomegaly; microcephaly; mental retardation; meningo-encephalitis; radiolucent bone disease; jaundice with onset within 24 hours of birth. 
A laboratory-confirmed CRS case is an infant with a positive blood test for rubella IgM who has clinicallyconfirmed CRS. An infant with a positive blood test for rubella IgM who does not have clinically confirmed CRS is classified as having congenital rubella infection ${ }^{18}$.

The surveillance and laboratory information which is captured using a standard case investigation form is later entered into a computerized database at each sentinel site, and is shared with WHO on a monthly basis. We reviewed the sentinel surveillance data submitted from the 4 countries (Burkina Faso, Rwanda, Zimbabwe, Zambia, Tanzania) that had regularly shared data with the WHO. We used descriptive analysis to characterize the demographic and clinical characteristics of the cases.

\section{Results}

A total of 383 suspected cases of CRS were notified from the 5 countries as of December 2016. Tanzania reported 152 suspected cases while 143 were reported from Zimbabwe. Fifty five percent of the suspected cases (211 out of 383) were classified as discarded (not CRS cases) as per the case classification algorithm, while 52 were laboratory confirmed and 67 were confirmed on clinical grounds. (Table 1)

Table 1. Classification of suspected cases of CRS by country. 2012 - 2016.

\begin{tabular}{|l|c|c|c|c|c|c|}
\hline & $\begin{array}{c}\text { Clinically } \\
\text { confirmed } \\
\text { CRS }\end{array}$ & $\begin{array}{c}\text { Lab } \\
\text { confirmed } \\
\text { CRS }\end{array}$ & $\begin{array}{c}\text { Congenital } \\
\text { Rubella } \\
\text { Infection }\end{array}$ & Discarded & $\begin{array}{c}\text { Not } \\
\text { classified }\end{array}$ & TOTAL \\
\hline $\begin{array}{l}\text { Burkina } \\
\text { Faso }\end{array}$ & 0 & 0 & 0 & 28 & 41 & 69 \\
\hline Rwanda & 0 & 0 & 0 & 5 & 0 & 5 \\
\hline Tanzania & 48 & 17 & 0 & 83 & 4 & 152 \\
\hline Zambia & 3 & 3 & 0 & 0 & 8 & 14 \\
\hline Zimbabwe & 16 & 32 & 0 & 95 & 0 & 143 \\
\hline TOTAL & 67 & 52 & 0 & 211 & 53 & 383 \\
\hline
\end{tabular}

The clinical picture of the suspected cases indicates that $51 \%$ were reported with a congenital heart disease, while 22 had cataracts. Hearing impairment was reported in only $5 \%$ of the suspected cases. Among the group B clinical signs, microcephaly was reported in $32 \%$ and neonatal

Table 2. Clinical signs in 383 suspected CRS cases. 2012 - 2016. Sentinel sites in 5 countries in AFR.

\begin{tabular}{|l|l|c|c|}
\hline \multicolumn{1}{|c|}{ Clinical sign } & $\begin{array}{c}\text { Number of } \\
\text { cases }\end{array}$ & $\%$ cases \\
\hline \multirow{5}{*}{ Group A } & Congenital Heart Disease & 195 & $51 \%$ \\
\cline { 2 - 4 } & Cataracts & 83 & $22 \%$ \\
\cline { 2 - 4 } & Pigmentary retinopathy & 26 & $7 \%$ \\
\cline { 2 - 4 } & Glaucoma & 22 & $6 \%$ \\
\cline { 2 - 4 } & Hearing impairment & 20 & $5 \%$ \\
\hline \multirow{5}{*}{ Group B } & Microcephaly & 121 & $32 \%$ \\
\cline { 2 - 4 } & Jaundice & 113 & $30 \%$ \\
\cline { 2 - 4 } & Splenomegaly & 80 & $21 \%$ \\
\cline { 2 - 4 } & Purpura & 54 & $14 \%$ \\
\cline { 2 - 4 } & Mental retardation & 16 & $4 \%$ \\
\cline { 2 - 4 } & Radiolucent bone disease & 10 & $2 \%$ \\
\cline { 2 - 4 } & Meningo-encephalitis & 7 & \\
\hline
\end{tabular}

jaundice in 30\%. (Table 2). A history of maternal febrile rash during pregnancy was elicited in only $41(10.7 \%)$ of the suspected cases.

Table 3. Confirmed cases of CRS by country by year.

\begin{tabular}{|l|c|c|c|c|c|c|}
\hline & 2012 & 2013 & 2014 & 2015 & 2016 & TOTAL \\
\hline Burkina Faso & & & & 0 & 0 & 0 \\
\hline Rwanda & & & & 0 & 0 & 0 \\
\hline Tanzania & & & 20 & 44 & 1 & 65 \\
\hline Zambia & & & 1 & 5 & 0 & 6 \\
\hline Zimbabwe & 14 & 6 & 2 & 20 & 6 & 48 \\
\hline Total & 14 & 6 & 23 & 69 & 7 & 119 \\
\hline
\end{tabular}

Out of the 119 confirmed CRS cases, 65 were from Tanzania and 48 were reported from Zimbabwe, while Burkina Faso and Rwanda did not have any confirmed case. (Table 3) Burkina Faso did not classify 41 of the reported 69 cases, and these make up the majority of the 53 cases that were not classified at all in the databases from the respective sentinel surveillance sites. (Table 1) The majority (43\%) of confirmed CRS cases were in the age group 6 - 11 months, while 21 were newborns. (Table 4)

Table 4. Confirmed cases of CRS by age group. 2012 - 2016. Sentinel surveillance in 5 countries.

\begin{tabular}{|l|c|c|c|c|c|c|}
\hline & $\begin{array}{c}0-28 \\
\text { days }\end{array}$ & $\begin{array}{c}1-5 \\
\text { months }\end{array}$ & $\begin{array}{c}6-11 \\
\text { months }\end{array}$ & $\begin{array}{c}1 \text { year or } \\
\text { more of } \\
\text { age }\end{array}$ & $\begin{array}{c}\text { Age } \\
\text { missing }\end{array}$ & Total \\
\hline $\begin{array}{l}\text { Burkina } \\
\text { Faso }\end{array}$ & 0 & 0 & 0 & 0 & 0 & 0 \\
\hline Rwanda & 0 & 0 & 0 & 0 & 0 & 0 \\
\hline Tanzania & 5 & 15 & 44 & 0 & 1 & 65 \\
\hline Zambia & 2 & 0 & 3 & 0 & 1 & 6 \\
\hline Zimbabwe & 14 & 20 & 4 & 2 & 8 & 48 \\
\hline Total & 21 & 35 & 51 & 2 & 10 & 119 \\
\hline
\end{tabular}

The most common major clinical manifestation (Group A) among the confirmed cases is congenital heart disease (72\%) followed by cataracts (32\%) and glaucoma (10\%). Among the Group B manifestations, microcephaly and splenomegaly were detected in $34 \%$ and $25 \%$ of the confirmed cases respectively. (Table 5)

Table 5. Clinical presentation in the 119 confirmed cases of CRS in 5 countries. $2012-2016$.

\begin{tabular}{|c|l|c|c|}
\hline \multicolumn{1}{|c|}{ Clinical sign } & $\begin{array}{c}\text { Number of } \\
\text { cases }\end{array}$ & $\%$ cases \\
\hline \multirow{4}{*}{ Group A } & Congenital Heart Disease & 86 & $72 \%$ \\
\cline { 2 - 4 } & Cataracts & 38 & $32 \%$ \\
\cline { 2 - 4 } & Glaucoma & 12 & $10 \%$ \\
\cline { 2 - 4 } & Hearing impairment & 6 & $5 \%$ \\
\cline { 2 - 4 } & Pigmentary retinopathy & 1 & $1 \%$ \\
\hline \multirow{5}{*}{ Group B } & Microcephaly & 40 & $34 \%$ \\
\cline { 2 - 4 } & Splenomegaly & 30 & $25 \%$ \\
\cline { 2 - 4 } & Jaundice & 16 & $13 \%$ \\
\cline { 2 - 4 } & Purpura & 14 & $8 \%$ \\
\cline { 2 - 4 } & Mental retardation & 10 & $3 \%$ \\
\cline { 2 - 4 } & Meningo-encephalitis & 4 & $0 \%$ \\
\cline { 2 - 4 } & Radiolucent bone disease & 0 & \\
\hline
\end{tabular}




\section{Discussion}

Zimbabwe established sentinel CRS surveillance in 2011 while Zambia and Tanzania started implementing it in 2014, and Rwanda and Burkina Faso in 2015. The number of years of reporting from these sentinel sites is too short to describe trends in CRS occurrence across the years. However, the limited surveillance data has yielded comparable information with other developing countries prior to introduction of rubella vaccine.

In this series, hearing impairment was detected in only $5 \%$ of the suspected cases. This is an indication of the difficulty to diagnose the condition in early infancy. Half of the suspected cases (51\%) and $72 \%$ of the confirmed cases presented with congenital heath disease, since the sentinel sites were mostly cardiac disease follow-up clinics in tertiary care centers. Cataracts were detected in $22 \%$ of the suspected cases and in a third of the confirmed CRS cases in this series. In the Sudan, among 31 suspected CRS cases under 1 year of age, 11 cases were serologically confirmed, with cataracts $(81.8 \%)$ and cardiac disease $(72.7 \%)$ being the most common clinical signs ${ }^{23}$.

In countries implementing rubella and CRS elimination activities, WHO recommends to establish febrile rash illness surveillance integrated with the surveillance for measles, along with the investigation of rubella outbreaks. In addition, WHO recommends the conduct of active surveillance for CRS following rubella outbreaks to detect and confirm suspected cases in the infant cohorts ${ }^{22,24}$. In the case of these 5 countries in our study, the timing and location of the sentinel sites did not include considerations to the presence of rubella epidemics. In Vietnam, CRS was diagnosed in 292 infants following a rubella epidemic, and the most common clinical symptoms were congenital heart disease (91.4\%), cataract(s) (45.2\%), splenomegaly (38.7\%) and purpura $(32.9 \%)^{25}$. In the Solomon Islands, following a rubella outbreak in May 2012, prospective CRS surveillance done in various clinical settings over a 4 months period in 2013 and helped to identify 13 CRS cases $^{26}$.

The CRS sentinel surveillance protocol indicates that the target age group for prospective CRS is infants less than one year of age because of the reliance on IgM testing, and the eventual decline in IgM levels. Although IgM antibodies may persist for up to 1 year, the rate of positivity declines after 6 months of life. Ideally, laboratory confirmation of CRS in an infant aged over six months should not rely on the IgM test alone if the result is negative. In such cases, serial IgG testing should also be included to check for a sustained level of antibody over several months ${ }^{18,22}$. In this series, children older than 12 months were included in the reporting from Zimbabwe, and there were cases with missing information on age, indicating the need to ensure that the protocols are strictly followed.
There is also the need to document the viral strains of detected rubella viruses. Molecular epidemiology that allows the differentiation of circulating rubella viruses, can be used to monitor the transmission pathways and to identify interruption of endemic virus transmission. There is a gap in the African Region with regards to the baseline data on circulating viruses ${ }^{27}$. As of 2015, only Algeria and DR Congo had documented rubella genotype data from among the 47 countries in the Region ${ }^{28}$. Unfortunately, none of the 5 countries reporting from the sentinel surveillance sites had documented the strains of rubella viruses from the lab confirmed CRS cases.

\section{Conclusion and recommendations}

In conclusion, the sentinel surveillance sites have managed to document suspected and confirmed cases of CRS within a short period of time, indicating that sensitive sentinel surveillance systems can play a role in the decision to introduce rubella vaccine and monitor the impact thereafter. As more and more countries prepare to introduce rubella vaccine into their immunisation programs, there is a need to ensure that all rubella outbreaks are thoroughly investigated and documented, to expand sentinel surveillance for CRS in more countries in the Region, to institute a mechanism to assure high sensitivity of case detection, and to complement this with retrospective record reviews for CRS cases in selected countries.

\section{Limitations}

This analysis does not take into account the rubella incidence in the countries at the time. It covers only 5 countries, and sentinel surveillance was operational for no more than 3 years except in the case of Zimbabwe. Therefore the findings may not be representative of the overall trends in the countries, and of the epidemiological situation in other countries in the subregion. The identification of the sentinel surveillance sites was done at country level, depending on the presence of paediatric tertiary care and specialty centers in each country, and the availability of existing working arrangements with the institutions. Even though the case definitions and case investigation were standardised, currently there is no mechanism to assure that all suspected cases are captured through the sentinel surveillance system, with the possibility that milder cases may have escaped detection.

\section{Acknowledgements}

We would like to thank the focal persons of the various sentinel surveillance sites and the laboratory staff involved in the investigation of suspected CRS cases.

\section{Conflict of Interest}

\section{None}




\section{References}

1. Mason WH, Rubella, Kliegman RM, et al. Eds. Nelson Textbook of Pediatrics, 19th Edition. 2011; P 1075 - 1078.

2. Lambert N, Strebel P, Orenstein W, et al. Rubella. Lancet. 2015; 385: 2297-307.

3. Banatvala JE, Brown DW. Rubella. Lancet. 2004; 363: 1127-37.

4. World Health Organisation. Manual for the laboratory diagnosis of measles and rubella virus infection. Second edition. August 2007. WHO/IVB/07.01.

5. World Health Organization. Introducing rubella vaccine into national immunization programmes: a step by step guide. 2015. WHO/ IVB/15.07/

6. Nsambu MN, Coulibaly T, Donnen P, et al. Fréquence de la rubéole à Kinshasa de 2010 à 2012, République Démocratique Du Congo: données issues du système de surveillance de la rougeole. Santé publique. Volume 26 / N³: 393- 397.

7. Goodson JL, Masresha B, Dosseh A, et al. Rubella Epidemiology in Africa in the Prevaccine Era, 2002-2009. J Infect Dis. 2011; 203: S215S225.

8. Mitiku K, Bedada T, Masresha B, et al. The Epidemiology of Rubella Disease in Ethiopia: Data From the Measles Case-based Surveillance System. J Infect Dis. 2011; 203: S239-S242.

9. Chimhuya S, Manangazira P, Mukaratirwa A, et al. Trends of rubella incidence during a 5-year period of case based surveillance in Zimbabwe. BMC Public Health. 2015; 15: 294. DOI 10.1186/s12889015-1642-4

10. Farra A, Pagonendji M, Manikariza A, et al. Epidemiology of primary rubella infection in the Central African Republic: data from measles surveillance, 2007-2014. BMC Infectious Diseases. 2016; 16: 505. DOI 10.1186/s12879-016-1842-2.

11. Tahita MC, Hübschen JM, Tarnagda Z, et al. Rubella seroprevalence among pregnant women in Burkina Faso. BMC Infectious Diseases. 2013; 13: 164. https://doi.org/10.1186/1471-2334-13-164]

12. Jonas A, Cardemil CV, Beukes A, et al. Rubella immunity among pregnant women aged 15-44 years, Namibia, 2010. Int J Infect Dis. 2016; 49: 196-201.

13. WHO Regional Committee for Africa. Measles Elimination by 2020 A Strategy for the African Region. AFR/RC61/R1. Available at: http://www.afro.who.int/en/sixty-first-session.html Last accessed February 2017.

14. World Health Organisation, Regional office for Africa. Regional Strategic Plan for Immunization 2014-2020. Brazzaville Congo: 2015.

15. World Health Organisation. Position paper on rubella vaccine. Wkly Epidemiol Rec No 29. 2011; 86: 301-316.
16. GAVI support for measles and measles-rubella vaccine. http://www.gavi. org/support/nvs/measles-rubella/ last accessed on 14 Sept 2017.

17. Rubella vaccine introduction in AFR. In "Progress with measles elimination in the WHO African region". Paper presented to the Mid Term Review of the Regional Strategic Plan for Immunisation. Sept 2017.

18. World Health Organization. Regional Office for Africa. African Regional guidelines for measles and rubella surveillance- Revised April 2015. Available at http://www.afro.who.int/index.php?option=com docman\&task=doc_download\&gid=10814\&Itemid=2593, Last accessed 17 April 2017.

19. Annual reports of CRS cases to the WHO, by country by year. http://apps.who.int/immunization_monitoring/globalsummary/ timeseries/tsincidencecrs.html last accessed on Sept 16, 2017.

20. Emilia Vynnycky, Elisabeth J Adams, Felicity $\mathrm{T}$ Cutts, et al. Seroprevalence and Immunisation Using Coverage Data to Estimate the Global Burden of Congenital Rubella Syndrome, 1996-2010: A Systematic Review. PLoS ONE. 2016; 11(3). e0149160. doi:10.1371/ journal. one. 0149160

21. Alleman MM, Wannemuehler KA, Hao L, et al. Estimating the burden of rubella virus infection and congenital rubella syndrome through a rubella immunity assessment among pregnant women in the Democratic Republic of the Congo: Potential impact on vaccination policy. Vaccine. 2016; 34: 6502-6511. http://dx.doi.org/10.1016/j. vaccine.2016.10.059

22. World Health Organisation. WHO-recommended standards for surveillance of selected vaccine-preventable diseases. Revised July 2008. WHO/V\&B/03.01 http://apps.who.int/iris/ bitstream/10665/68334/1/WHO_V-B_03.01_eng.pdf last accessed 17 Sept 2017.

23. Adam 0, El Hussein A, El Eragi A, et al. Primary investigation of 31 infants with suspected congenital rubella syndrome in Sudan. Clin Microbiol Infect. 2010;16(6):678-82.

24. Kyaw Zin Thant. Active surveillance for congenital rubella syndrome in Myanmar: Bull WHO. 2006; 84: 12 - 20

25. Thant KZ, Oo WM, Myint TT, et al. Congenital rubella syndrome (CRS) in Vietnam 2011-2012: CRS epidemic after rubella epidemic in 20102011. Vaccine. 2015; 33: 3673-3677. http://dx.doi.org/10.1016/j. vaccine.2015.06.035

26. Durski KN, Tituli C, Ogaoga D, et al. An outbreak investigation of congenital rubella syndrome in Solomon Islands, 2013. Western Pac Surveill Response J. 2016; 7(1): 10-13.

27. Abernathy ES, Hübschen JM, Muller CP, et al. Status of Global Virologic Surveillance for Rubella Viruses. J Infect Dis. 2011; 204: S524-S532.

28. Mulders MN, Rota PA, Icenogle JP, et al. Global measles and rubella laboratory network support for elimination goals, 2010-2015. Wkly Epidemiol rec. 2016; No 18, 91: 237-248. 